\title{
Crescimento e sobrevivência da tilápia chitralada submetida a diferentes fotoperíodos
}

\author{
Katia Santos Bezerra ${ }^{(1)}$, Athiê Jorge Guerra Santos ${ }^{(1)}$, Maxwel Rozendo Leite $^{(1)}$, Alexandre Magnun da Silva ${ }^{(1)}$ \\ e Misleni Ricarte de Lima ${ }^{(1)}$
}

\begin{abstract}
(1)Universidade Federal Rural de Pernambuco, Departamento de Pesca e Aqüicultura, Avenida Dom Manuel de Medeiros, s/noo, Dois Irmãos, CEP 52171-900 Recife, PE. E-mail: katia00sb@hotmail.com, ajgs@depaq.ufrpe.br, maxcyclop@hotmail.com, alexandre_recife@hotmail.com, misleniricarte@hotmail.com
\end{abstract}

\begin{abstract}
Resumo - O objetivo deste trabalho foi avaliar o efeito do fotoperíodo no crescimento e sobrevivência da tilápia chitralada, Oreochromis niloticus, durante e após a fase de reversão sexual. O trabalho foi realizado em 2006 e 2007, em dois experimentos. No primeiro experimento, foram utilizadas pós-larvas de $0,031 \pm 0,0058 \mathrm{~g} \mathrm{e} 1,24 \pm 0,096 \mathrm{~cm}$ à densidade de 1,5 pós-larva $\mathrm{L}^{-1}$. As pós-larvas foram submetidas aos fotoperíodos $0,8,12,16,24$ horas de luz e a 12,5 horas de luz natural, durante 28 dias. Os animais foram alimentados com ração de $45 \%$ de proteína bruta, com hormônio andrógeno, na proporção de $20 \%$ da biomassa total, dividida em quatro refeições. As biometrias foram realizadas no início e no fim do experimento. No segundo experimento, foram utilizados alevinos de $0,38 \pm 0,16 \mathrm{~g}$ e $2,81 \pm 0,40 \mathrm{~cm}$, revertidos para macho. $\mathrm{O}$ desenho experimental foi semelhante ao do primeiro experimento, exceto a densidade de estocagem $\left(0,33\right.$ peixe $\left.\mathrm{L}^{-1}\right)$, a taxa de alimentação ( $10 \%$ da biomassa total) e o tempo de experimento ( 46 dias). Realizaram-se biometrias quinzenais. $\mathrm{O}$ fotoperíodo interferiu mais expressivamente na sobrevivência das pós-larvas da tilápia, e a luz natural foi a mais indicada durante a reversão sexual. A melhor sobrevivência dos alevinos ocorreu no grupo 0 hora de luz. Melhores crescimentos em pesos e comprimentos, porém, aconteceram nos demais fotoperíodos. Taxas de sobrevivência e de crescimento, em resposta a diferentes fotoperíodos, parecem estar relacionadas à idade do peixe.
\end{abstract}

Termos para indexação: Oreochromis niloticus, crescimento, período de luz, sobrevivência.

\section{Growth and survival of tilapia chitralada submitted to different photoperiods}

Abstract - The objective of this work was to assess the effect of photoperiod in the growth and survival of tilapia chitralada, Oreochromis niloticus, during and after the sexual reversion. The work was carried out in 2006 and 2007, and divided into two experiments. In the first experiment, post-larvae of $0.031 \pm 0.0058 \mathrm{~g}$ and $1.24 \pm 0.096 \mathrm{~cm}$ were used, at the density of 1.5 post-larva $\mathrm{L}^{-1}$. The post-larvae were submitted to the following photoperiods: 0 , $8,12,16,24$ hours of light and 12.5 hours of natural light, during 28 days. The fishes were fed with $45 \%$ crude protein balanced food, containing androgen hormone, at $20 \%$ of the total biomass, divided into four meals. Sampling was done at the beginning and at the end of the experiment. In the second experiment, sex reverted fingerlings of $0.38 \pm 0.16 \mathrm{~g}$ and $2.81 \pm 0.40 \mathrm{~cm}$ were used. The experiment protocols were the same as in the former experiment, except for the stock density $\left(0.33\right.$ fish L $\left.^{-1}\right)$, food ratio $(10 \%$ of the total biomass $)$ and the experimental period (46 days). Sampling was done every fifteen days. The photoperiod interfered more intensively in the survival of the tilapia post-larvae, and the natural light was the recommended photoperiod during the sexual reversion. The best survival rate of the fingerlings occurred in the 0-hour light group; regarding fingerling growth, however, better results in bodyweight and length were found in the other photoperiod groups. It seems that the photoperiod responses to survival and growth are related to fish age.

Index terms: Oreochromis niloticus, growth, light period, survival.

\section{Introdução}

A aqüicultura é uma das atividades com maior representatividade na produção de proteína de origem animal, que gera receita de milhões de dólares para vários países. Desde 1970, esta atividade vem crescendo à taxa média de $9,2 \%$ ao ano e superando a produção de outros animais, que cresce apenas 2,8\%, tornando-se, assim, uma das atividades econômicas de maior crescimento (Assad, 2004).

A produção de tilápias no mundo alcançou 2.025.560 Mg em 2005, e foi responsável por 6,7\% da 
produção global de peixes cultivados. O Brasil está em sexto lugar na escala mundial dos maiores produtores de tilápia, responsável por 3,3\% do total da produção (FAO, 2007).

Segundo Kubitza (2000), o sucesso das tilápias devese à sua rusticidade, bom desenvolvimento corporal, facilidade de reprodução, excelentes características organolépticas, ausência de espinhos intermusculares em forma de $\mathrm{Y}$ e menor tempo para atingir o tamanho comercial. Entre as espécies cultivadas no Brasil, a tilápia-do-nilo Oreochromis niloticus, linhagem chitralada, é a mais utilizada na produção comercial. De acordo com Zimmermann (2000), essa variedade destaca-se por apresentar desempenho de crescimento superior em relação às outras espécies de tilápia. Esse peixe foi introduzido no Brasil em 1996, oriundo do Asian Institute of Technology (AIT) e, nos últimos anos, vem passando por melhoramentos genéticos.

A demanda de peixes em nosso país vem aumentando progressivamente, com obtenção de alevinos de boa qualidade como parte fundamental no processo de produção. A larvicultura corresponde ao período mais importante dentro da cadeia produtiva, pois é a partir do bom gerenciamento dessa fase que se obtêm os melhores índices de qualidade e produção animal, desejados nas fases posteriores (Meurer et al., 2005).

O processo da reversão sexual é praticado, quando um dos sexos possui marcada superioridade na taxa de crescimento em relação ao outro, além de ser vantajoso no controle da reprodução, na contenção de gastos energéticos na reprodução, uniformidade de tamanho e na redução dos efeitos da maturação sexual sobre a aparência e a qualidade da carne (Beardmore et al., 2001). O fotoperíodo também influencia o desenvolvimento e a sobrevivência, em suas diferentes fases ontogênicas, pois a luz auxilia tanto na estratégia alimentar como no estímulo a outras atividades metabólicas de várias espécies de peixes (ReynalteTataje, 2002). O fotoperíodo corresponde a um dos diversos estímulos ambientais e está relacionado com a duração do tempo de luz ao longo de um dia. A intensidade e aumento desse tempo de luz se modificam com as estações do ano e o clima da região (Bromage et al., 2001).

A maioria das larviculturas nacionais opera com a produção de alevinos de tilápias revertidas sexualmente para macho, a fim de se obterem peixes que cresçam mais rápido em pouco tempo. Sabe-se que o fotoperíodo influencia no crescimento e no ciclo reprodutivo da tilápia (Bromage et al., 2001). Contudo, existem poucas informações sobre os efeitos desse fator ecológico no desempenho da tilápia chitralada, nas fases iniciais do seu crescimento e, em especial, durante e após o processo de reversão sexual.

O objetivo deste trabalho foi avaliar o efeito do fotoperíodo no crescimento e na sobrevivência da tilápia, da linhagem chitralada, durante e após a fase de reversão sexual.

\section{Material e Métodos}

O estudo foi realizado na Estação de Aqüicultura Continental Prof. Johei Koike, do Departamento de Pesca e Aqüicultura da Universidade Federal Rural de Pernambuco, entre dezembro de 2006 e junho de 2007. As tilápias da linhagem chitralada foram adquiridas na Estação e na Empresa Mar Doce do Nordeste Piscicultura e Projeto Ltda. No início de cada experimento, os peixes foram mensurados (peso e comprimento) e tratados profilaticamente com parasiticida Labcon Ictio. Após 24 horas, os peixes foram acondicionados e distribuídos nos aquários experimentais, em delineamento inteiramente ao acaso, com seis tratamentos e duas repetições.

No primeiro experimento, avaliou-se o efeito do fotoperíodo em pós-larvas, durante a reversão sexual. As pós-larvas ( $\mathrm{n}=611,0,031 \pm 0,0058 \mathrm{~g}$ e 1,24 $\pm 0,096 \mathrm{~cm})$ foram submetidas a seis diferentes fotoperíodos: 0,8 , 12, 16, 24 horas de luz e controle (luz natural, 12,5 horas de luz), com duas repetições. A densidade de estocagem foi de 1,5 pós-larva $\mathrm{L}^{-1}$. Sobre cada repetição, instalou-se um sistema de lâmpadas fluorescentes ( $40 \mathrm{~W}$ ), apoiado sobre os aquários experimentais cobertos com lonas de plástico. Os fotoperíodos foram controlados por meio de equipamento "multi-timer", tipo Gubintec TI-12A.

A água foi renovada durante sete horas diárias, em vazão correspondente a duas trocas do volume do aquário por dia. Para o escoamento da água, se utilizou tubo de PVC $(20 \mathrm{~mm})$ em forma de sifão. Na região externa do sifão, acoplaram-se mangueiras ( 0,5 polegada) que finalizavam num cano central de escoamento.

O controle dos níveis de oxigênio dissolvido foi realizado por aeração contínua. A limpeza dos aquários foi realizada duas vezes ao dia, e estes foram esterilizados semanalmente com sal grosso não iodado $\left(3 \mathrm{~g} \mathrm{~L}^{-1}\right)$. 
As pós-larvas foram alimentadas com ração comercial, cujos níveis de garantia de constituição são: $45 \%$ de proteína bruta, com $60 \mathrm{mg} \mathrm{kg}^{-1}$ do hormônio 17-alfa-metil-testosterona, $13 \%$ de umidade (máximo), $8 \%$ de extrato etéreo (mínimo), $7 \%$ de fibra (máximo), $14 \%$ de cinzas (máximo), 2,5\% de Ca (máximo), 0,6\% de $\mathrm{P}$ (mínimo), 12\% de mineral (máximo), $50 \mathrm{mg} \mathrm{kg}^{-1} \mathrm{de} \mathrm{Fe}$, $10 \mathrm{mg} \mathrm{kg}^{-1} \mathrm{de}$ Co, $100 \mathrm{mg} \mathrm{kg}^{-1} \mathrm{de} \mathrm{Zn,} 50 \mathrm{mg} \mathrm{kg}^{-1} \mathrm{de} \mathrm{Mn}$, $1 \mathrm{mg} \mathrm{kg}^{-1}$ de I, $0,15 \mathrm{mg} \mathrm{kg}^{-1}$ de Se, 10.000 UI de vitamina $A, 2.500$ UI de vitamina $D, 200$ UI de vitamina $\mathrm{E}, 30 \mathrm{mg} \mathrm{kg}^{-1}$ de vitamina $\mathrm{K}, 10 \mathrm{mg} \mathrm{kg}^{-1} \mathrm{de}$ ácido fólico, $0,60 \mathrm{mg} \mathrm{kg}^{-1}$ de biotina, $1.400 \mathrm{mg} \mathrm{kg}^{-1} \mathrm{de}$ colina, $100 \mathrm{mg} \mathrm{kg}^{-1}$ de ácido nicotínico, $50 \mathrm{mg} \mathrm{kg}^{-1} \mathrm{de}$ ácido pantotênico, $30 \mathrm{mg} \mathrm{kg}^{-1}$ de tiamina, $30 \mathrm{mg} \mathrm{kg}^{-1} \mathrm{de}$ riboflavina, $30 \mathrm{mg} \mathrm{kg}^{-1}$ de piridoxina, $30 \mathrm{mg} \mathrm{kg}^{-1} \mathrm{de}$ vitamina B12, $200 \mathrm{mg} \mathrm{kg}^{-1}$ de vitamina Ce $100 \mathrm{mg} \mathrm{kg}^{-1} \mathrm{de}$ antioxidante. A quantidade de ração ofertada foi $20 \%$ da biomassa total, distribuída em quatro refeições, às 10 , 12,14 e 16h. A biometria (peso e comprimento) foi realizada no início e no fim do experimento, aos 28 dias.

No segundo experimento, foram avaliados os efeitos do fotoperíodo em alevinos revertidos para macho. Utilizaram-se 183 alevinos $(0,38 \pm 0,16 \mathrm{~g} \mathrm{e} 2,81 \pm 0,40 \mathrm{~cm})$. $\mathrm{O}$ delineamento experimental foi semelhante ao do primeiro experimento, exceto quanto à densidade de estocagem, que foi 0,33 indivíduos $\mathrm{L}^{-1}$. Os alevinos foram aclimatados por cinco dias em cada regime de fotoperíodo, antes do início do experimento.

Foi oferecida ração comercial em pó com $45 \%$ de proteína bruta, durante 30 dias, tendo-se aumentado gradativamente a granulometria até $3 \mathrm{~mm}$, que foi fornecida por mais 16 dias. A biometria (peso e comprimento) foi realizada a cada 15 dias, a fim de se acompanhar o crescimento e corrigir a alimentação, calculada em $10 \%$ da biomassa total. O experimento teve a duração de 46 dias.

A sobrevivência foi avaliada ao final de cada experimento. Os dados de crescimento em peso e comprimento foram expressos por $\mathrm{Wt}=\Phi \times \mathrm{Lt} \mathrm{t}^{\theta}$, em que Wt é o peso total, Lt é o comprimento total, $\Phi$ é o fator de condição, $\theta$ é a constante; o coeficiente de variação é $\mathrm{CV}(\%)=(100 \times \mathrm{S}) / \overline{\mathrm{X}}$; a taxa de crescimento relativo é TCR $=\left(\mathrm{W}_{\mathrm{F}} / \mathrm{W}_{\mathrm{i}}\right)$, em que $\mathrm{W}_{\mathrm{f}}$ é o peso final e $\mathrm{W}_{\mathrm{i}}$ é o peso inicial; a conversão alimentar aparente $(\mathrm{CAA})=$ quantidade de ração fornecida $(\mathrm{kg}) / \mathrm{ganho}$ de biomassa; e a sobrevivência é $\mathrm{S}=100\left(\mathrm{n}^{\mathrm{o}}\right.$ indivíduos inicial)/no indivíduos final. Os dados foram analisados conforme Mendes (1999).
Os dados foram submetidos à análise de variância (ANOVA), e as médias foram comparadas pelo teste de Tukey, a 5\% de probabilidade (Mendes, 1999; Aayres et al., 2003).

\section{Resultados e Discussão}

Ao final do experimento, as médias de peso para os peixes dos tratamentos 8 e 24 horas de luz foram diferentes dos demais grupos e entre si (Tabela 1). Quanto ao comprimento médio final, não houve diferença significativa entre os tratamentos, exceto quanto ao tratamento 24 horas de luz $(3,47 \mathrm{~cm})$. A menor sobrevivência (11\%) ocorreu no grupo 24 horas de luz, e a maior $(77,44 \%)$ no grupo controle.

A resposta aos fotoperíodos pode estar relacionada à idade do peixe. As pós-larvas foram mais sensíveis aos diferentes fotoperíodos, principalmente quanto à sobrevivência. Observou-se agitação constante nas pós-larvas do grupo 24 horas de luz até o 15 dia, quando houve mortalidade de $89 \%$, provavelmente em conseqüência do estresse, ocasionado pelo excesso de luz. Segundo Pezzato et al. (2004), uma série de mudanças fisiológicas e bioquímicas, mediadas por ação nervosa e hormonal, ocorre nos peixes quando uma exaustão adaptativa é de um modo geral alcançada. Os animais se tornam altamente vulneráveis a variações ambientais e a infecções. Almazán-Rueda et al. (2005) observaram estresse e agressividade no "catfish" africano, Clarias gariepinus, submetido a 24 horas de luz. De acordo com os autores, boa parte desses indivíduos chegou ao seu limiar máximo de suporte ao estresse, por excesso de luz, e uma leve mudança dos parâmetros físico-químicos do ambiente foi o suficiente para causar o estresse, a agressividade e a morte. Este fato também foi observado por Imsland et al. (1995). Espécies de peixes de água doce parecem ser mais sensíveis à variação de fotoperíodo do que espécies diádromas marinhas, no entanto, estudos sobre respostas aos fotoperíodos com peixes de água doce ainda são bastante escassos (El-Sayed \& Kawanna, 2004).

Sigholt et al. (1995) também observaram baixa sobrevivência de salmão-do-atlântico (Salmo salar), na fase inicial de vida e quando submetido à luz contínua. $\mathrm{O}$ crescimento, porém, foi similar ao do fotoperíodo natural.

O crescimento dos peixes submetidos aos diferentes fotoperíodos, no presente trabalho, pouco variou em relação ao fotoperíodo natural, exceto o 
dos grupos 0 e 24 horas de luz. Resultados similares foram observados por Abdel-Fattah et al. (2004), que observaram que as respostas dos peixes ao fotoperíodo estão relacionadas com a idade do animal. Pode-se deduzir que a tilápia, em sua fase inicial de vida, e provavelmente outros peixes teleósteos, exibem pouca resposta ao fotoperíodo, principalmente quanto ao peso e comprimento. Segundo Barlow et al. (1995), fotoperíodos longos, durante a fase larval de Lates calcarifer (Bloch), melhoraram o crescimento, mas não a sobrevivência. Há concordância com os autores apenas quanto à sobrevivência, pois o crescimento dos indivíduos, no presente trabalho, pouco se diferenciou entre os tratamentos; e a segunda maior sobrevivência ocorreu em 16 horas de luz, que não diferiu do tratamento 0 hora de luz. Trotter et al. (2003) investigaram o efeito da luz em larvas de Latris lineata, e obtiveram o melhor crescimento nos fotoperíodos 18 e 24 horas de luz. Isso sugere que a influência do fotoperíodo, durante a fase de pós-larva, também pode variar de acordo com a espécie.

O "catfish" (Wallago attu, Bloch \& Schneider) na fase de pós-larva apresentou melhor ganho de peso no fotoperíodo 12 horas de luz (Giri et al., 2002), resultado diferente daquele obtido nas pós-larvas de tilápia do presente estudo, em que o melhor ganho de peso foi no tratamento 24 horas de luz. No entanto, o controle apresentou o melhor desempenho quanto à sobrevivência, faixa essa também recomendada por outros autores para a tilápia-do-nilo e "catfish"
Leiocassis longirostris (El-Sayed \& Kawanna, 2004; Han et al., 2005).

Quanto aos coeficientes de variação do peso e comprimento finais, os menores valores ocorreram no grupo 16 horas de luz, ou seja, 39,46 e 15,94\%, respectivamente, o que indica ser este o tratamento de maior homogeneidade. Os maiores valores foram encontrados nos grupos 24 horas de luz - 88,66 e 20,37\% - e 8 horas de luz - 67,21 e 19,94\% -, ou seja, menor homogeneidade.

A maior taxa de crescimento relativo (TCR) do peso e comprimento foi verificada no tratamento 24 horas de luz. Provavelmente, este resultado esteja relacionado à mortalidade de $89 \%$ no 15 o dia experimental, pois os peixes sobreviventes foram favorecidos por menor densidade, apesar de a ração ter sido reajustada à biomassa.

A conversão alimentar aparente não diferiu entre os tratamentos. El-Sayed \& Kawanna (2004) acompanharam, durante 90 dias, o desenvolvimento de pós-larvas $(0,02 \mathrm{~g})$ de tilápia-do-nilo, até a fase de alevinos $(2,4 \pm 0,05 \mathrm{~g})$, sob fotoperíodos de $6,12,18 \mathrm{e}$ 24 horas de luz, e registraram taxas de conversão alimentar de 1,85, 1,78, 1,55 e 1,52, respectivamente, ou seja, valores menores do que aqueles observados no presente trabalho. Essa diferença pode ser explicada pelo fato de o cálculo do arraçoamento ter sido feito com base em $20 \%$ da biomassa, valor estipulado no início do experimento, e provavelmente esteve superior à capacidade de conversão das pós-larvas de tilápia no presente trabalho.

Tabela 1. Desempenho de crescimento das pós-larvas de tilápia chitralada, durante a reversão sexual ${ }^{(1)}$.

\begin{tabular}{lcccccc}
\hline Variável & \multicolumn{5}{c}{ Fotoperíodos (horas de luz) } \\
\cline { 2 - 7 } & 0 & 8 & Controle (12,5) & 12 & 16 & 24 \\
\hline Densidade de estocagem (ind. L ${ }^{-1}$ ) & 1,50 & 1,50 & 1,50 & 1,50 & 1,50 & 1,50 \\
Número inicial de indivíduos & 200 & 118 & 130 & 200 & 200 & 200 \\
Peso médio inicial (g) & $0,03 \pm 0,006$ & $0,03 \pm 0,006$ & $0,03 \pm 0,006$ & $0,03 \pm 0,006$ & $0,03 \pm 0,006$ & $0,03 \pm 0,006$ \\
Peso médio final (g) & $0,37 \pm 0,258 \mathrm{a}$ & $0,48 \pm 0,313 \mathrm{~b}$ & $0,34 \pm 0,225 \mathrm{a}$ & $0,33 \pm 0,295 \mathrm{a}$ & $0,38 \pm 0,216 \mathrm{a}$ & $0,75 \pm 0,296 \mathrm{c}$ \\
Comprimento médio inicial (cm) & $1,24 \pm 0,096$ & $1,24 \pm 0,096$ & $1,24 \pm 0,096$ & $1,24 \pm 0,096$ & $1,24 \pm 0,096$ & $1,24 \pm 0,096$ \\
Comprimento médio final (cm) & $2,8 \pm 0,55 \mathrm{a}$ & $2,8 \pm 0,63 \mathrm{a}$ & $2,6 \pm 0,53 \mathrm{a}$ & $2,6 \pm 0,51 \mathrm{a}$ & $2,7 \pm 0,48 \mathrm{a}$ & $3,4 \pm 0,55 \mathrm{~b}$ \\
CV inicial peso (\%) & 19,07 & 19,07 & 19,07 & 19,07 & 19,07 & 19,07 \\
CV final peso (\%) & 67,72 & 67,21 & 57,54 & 65,61 & 39,46 & 88,66 \\
CV inicial comprimento (\%) & 7,80 & 7,80 & 7,80 & 7,80 & 7,80 & 7,80 \\
CV final comprimento (\%) & 19,94 & 20,37 & 17,62 & 22,46 & 15,94 & 19,37 \\
TCR peso (\%) & 1.177 & 1.538 & $1.081,42$ & $1.073,54$ & $1.209,78$ & $2.420,47$ \\
TCR comprimento (\%) & 226,35 & 230,05 & 211,12 & 212,72 & 223,50 & 280,83 \\
CAA (média) & 2,70 & 2,70 & 2,70 & 2,70 & 2,70 & 2,70 \\
Sobrevivência (\%) & $60,00 \mathrm{~b}$ & $49,15 \mathrm{c}$ & $77,44 \mathrm{a}$ & $44,00 \mathrm{c}$ & $62,50 \mathrm{~b}$ & $11,00 \mathrm{~d}$ \\
\hline
\end{tabular}

${ }^{(1)}$ Médias seguidas por letras iguais, na linha, não diferem entre si pelo teste de Tukey, a 5\% de probabilidade; TCR: taxa de crescimento relativo; CAA: conversão alimentar aparente. 
Quanto ao experimento com os alevinos, não houve diferença significativa para o peso médio entre os tratamentos $(p>0,05)$, exceto para o de 0 hora de luz, que apresentou os menores valores. Dados semelhantes foram verificados para o comprimento médio. A sobrevivência variou de $81 \%$ (controle) a 94\% (grupo 0 hora de luz).

Segundo Barlow et al. (1995), as taxas de crescimento em juvenis de Lates calcarifer (Bloch), com fotoperíodo natural e com aumento do tempo de luz, são quase sempre similares. Provavelmente, o fraco desempenho dos alevinos do grupo 0 hora de luz esteja relacionado à falta de ritmicidade alimentar, em razão da ausência de luz e do controle mais efetivo da glândula pineal, que restringiu apenas ao odor atrativo do alimento (Bromage et al., 2001; Biswas \& Takeuchi, 2002; Biswas \& Takeuchi, 2003).

Apesar de não se ter aferido a intensidade de luz, os valores de sobrevivência do grupo 12 horas de luz, observado no presente trabalho, foram similares aos obtidos por Han et al. (2005), quando estudaram o efeito da intensidade de luz (312 lux) e fotoperíodo (12 horas de luz), no crescimento de juvenis Leiocassis longirostris Günther. Segundo os seus resultados, houve interferência de tais fatores no aumento da sobrevivência e crescimento da referida espécie.

As pós-larvas foram mais sensíveis aos diferentes fotoperíodos do que os alevinos, principalmente quanto à sobrevivência. Conforme indicado na Tabela 2, a melhor taxa de sobrevivência ocorreu no grupo 0 hora de luz. O peso e crescimento médios dos alevinos neste grupo, porém, apresentou os piores resultados, tendo-se diferenciado estatisticamente dos demais fotoperíodos. Resultados similares foram obtidos com juvenis de L. longirostris, em que o crescimento foi significativamente reduzido, quando se baixou o tempo de exposição da luz, sem afetar, porém, a sobrevivência (Han et al., 2005). El-Sayed \& Kawanna (2004) obtiveram melhor crescimento nos fotoperíodos $24 \mathrm{e}$ 18 horas de luz para a tilápia-do-nilo. No entanto, não se diferenciaram significativamente, assim como foi observado no presente trabalho. Os autores ressaltaram, ainda, que a influência do fotoperíodo no crescimento da tilápia-do-nilo depende do estágio de desenvolvimento, e que pós-larvas são mais sensíveis às manipulações de fotoperíodos do que os alevinos. Rad et al. (2006) demonstraram que as respostas a fotoperíodos longos (24, 20 e 18 horas de luz) são mais evidenciadas a partir de 12 semanas experimentais, e obtiveram os melhores resultados no fotoperíodo 24 horas de luz, no final da 24a semana.

A partir dos dados do presente trabalho, pode-se deduzir que em estágios de desenvolvimento mais avançados da tilápia chitralada, o prolongamento do fotoperíodo tenha maior influência no crescimento, o que foi verificado por outros autores, com diferentes espécies (Sigholt et al., 1994; Downing \& Litvak, 2002; Trippel $\&$ Neil, 2003; Imsland et al., 2005).

Os resultados dos coeficientes de variação do peso $(42,51 \%)$ e comprimento $(14,73 \%)$ finais foram menores

Tabela 2. Desempenho de crescimento dos alevinos de tilápia chitralada, após a reversão sexual(1).

\begin{tabular}{|c|c|c|c|c|c|c|}
\hline \multirow[t]{2}{*}{ Variável } & \multicolumn{6}{|c|}{ Fotoperíodos (horas de luz) } \\
\hline & 0 & 8 & Controle $(12,5)$ & 12 & 16 & 24 \\
\hline Densidade de estocagem (ind. $\mathrm{L}^{-1}$ ) & 0,33 & 0,33 & 0,33 & 0,33 & 0,33 & 0,33 \\
\hline Número inicial de indivíduos & 16 & 40 & 27 & 40 & 20 & 20 \\
\hline Peso médio inicial (g) & $0,38 \pm 0,16$ & $0,38 \pm 0,16$ & $0,38 \pm 0,16$ & $0,38 \pm 0,16$ & $0,38 \pm 0,16$ & $0,38 \pm 0,16$ \\
\hline Peso médio final $(\mathrm{g})$ & $7,0 \pm 3,61 \mathrm{a}$ & $11,40 \pm 7,84 \mathrm{~b}$ & $12,00 \pm 5,75 b$ & $13,90 \pm 5,89 b$ & $15,70 \pm 7,00 \mathrm{~b}$ & $11,90 \pm 6,61 b$ \\
\hline Comprimento médio inicial $(\mathrm{cm})$ & $2,81 \pm 0,40$ & $2,81 \pm 0,40$ & $2,81 \pm 0,40$ & $2,81 \pm 0,40$ & $2,81 \pm 0,40$ & $2,81 \pm 0,40$ \\
\hline Comprimento médio final (cm) & $6,90 \pm 1,20 \mathrm{a}$ & $8,00 \pm 1,88 b$ & $8,20 \pm 1,38 b$ & $8,80 \pm 1,30 b$ & $9,00 \pm 1,49 b$ & $8,30 \pm 1,67 b$ \\
\hline CV inicial peso $(\%)$ & 43,29 & 43,29 & 43,29 & 43,29 & 43,29 & 43,29 \\
\hline CV final peso (\%) & 51,83 & 68,71 & 48,03 & 55,64 & 44,66 & 42,51 \\
\hline CV inicial comprimento (\%) & 14,48 & 14,48 & 14,48 & 14,48 & 14,48 & 14,48 \\
\hline CV final comprimento $(\%)$ & 17,40 & 23,43 & 16,97 & 20,21 & 16,60 & 14,73 \\
\hline TCR peso $(\%)$ & $200,00 \mathrm{a}$ & $203,60 a$ & $229,40 \mathrm{a}$ & $267,30 \mathrm{a}$ & $249,20 \mathrm{a}$ & $247,92 \mathrm{a}$ \\
\hline TCR comprimento (\%) & $121,05 \mathrm{a}$ & $123,10 \mathrm{a}$ & $125,57 \mathrm{a}$ & $137,5 \mathrm{a}$ & $130,43 a$ & $127,7 \mathrm{a}$ \\
\hline CAA (média) & 1,95 & 1,95 & 1,95 & 1,95 & 1,95 & 1,95 \\
\hline Sobrevivência (\%) & $94,00 \mathrm{a}$ & $85,00 \mathrm{~b}$ & $81,00 \mathrm{~b}$ & $85,00 \mathrm{~b}$ & $89,00 \mathrm{a}$ & $93,00 \mathrm{a}$ \\
\hline
\end{tabular}

${ }^{(1)}$ Médias seguidas por letras iguais, na linha, não diferem entre si pelo teste de Tukey, a 5\% de probabilidade; TCR: taxa de crescimento relativo; CAA: conversão alimentar aparente. 
no grupo 24 horas de luz. Os tratamentos 8 horas de luz $(68,71$ e $23,43 \%)$ e o controle $(48,03$ e $16,97 \%)$ apresentaram os maiores valores. Imsland et al. (2006) identificaram maior homogeneidade em juvenis de Hippoglossus hippoglossus no fotoperíodo natural. A menor variabilidade em peso do Clarias gariepinus, foi de $48 \%$, obtido no fotoperíodo 18 horas de luz (Almazán-Rueda et al., 2005). A tilápia chitralada apresentou maior homogeneidade de coeficiente de peso e comprimento, em comparação aos resultados de Almazán-Rueda et al. (2005).

Quanto à taxa de crescimento relativo do peso e comprimento, não houve diferença entre os tratamentos. Possivelmente, respostas significativas sejam mais observadas em estágios de desenvolvimento subseqüentes, assim como foi verificado no trabalho de El-Sayed \& Kawanna (2004).

Em relação à conversão alimentar aparente, observou-se igual valor médio - 1,95 - em todos os tratamentos. Biswas et al. (2005) investigaram o Pagrus major e demonstraram resultados similares aos obtidos no presente trabalho. As conversões foram de 1,95 para o fotoperíodo de 12 horas de luz, e 1,97 para o de 24 horas de luz. O peso inicial dos peixes, nesse experimento, variou entre 19 e $120 \mathrm{~g}$. Melhores resultados foram obtidos posteriormente, quando se variou o peso inicial e os fotoperíodos (Biswas et al., 2006). Numa criação experimental de 99 dias com o H. hippoglossus, com peso de 44,7 g no início do experimento, os autores observaram conversão alimentar de 0,64 para o fotoperíodo de 20 horas de luz e 0,72 para 24 horas de luz (Imsland et al., 2006). Provavelmente, essas diferenças na conversão alimentar estejam relacionadas, também, à espécie e à sua fase ontogênica no início do experimento.

\section{Conclusões}

1. O fotoperíodo natural é o mais indicado para a criação de tilápia chitralada, na fase de reversão sexual.

2. Fotoperíodos mais longos promovem maior crescimento dos alevinos de tilápia chitralada.

3. As respostas de sobrevivência e crescimento de tilápia chitralada a diferentes fotoperíodos estão relacionadas à idade dos peixes.

\section{Referências}

AAYRES, M.; AAYRES, J.; AAYRES, D.L.; SANTOS, A.S. Bioestat: versão 3.0. Belém: Sociedade Civil Mamirauá, 2003.
ALMAZÁN-RUEDA, P.; VAN HELMOND, A.T.M.; VERRETH, J.A.J.; SCHRAMA, J.W. Photoperiod affects growth, behavior and stress variables in Clarias gariepinus. Journal of Fish Biology, v.67, p.1029-1039, 2005.

ASSAD, L.T. Uma visão de futuro: aqüicultura e pesca no Brasil. Aqüicultura e Pesca, v.1, p.30, 2004.

BARLOW, C.G.; PEARCE, M.G.; RODGERS, L.J.; CLAYTON, P. Effects of photoperiod on growth, survival and feeding periodicity of larval and juvenile barramundi Lates calcarifer (Bloch). Aquaculture, v.138, p.159-168, 1995.

BEARDMORE, J.A.; MAIR, G.C.; LEWIS, R.I. Monosex male production in finfish as exemplified by tilapia: applications, problems, and prospects. Aquaculture, v.197, p.283-301, 2001.

BISWAS, A.K.; SEOKA, M.; INOUE, Y.; TAKII, K.; KUMAI, H. Photoperiod influences the growth, food intake, feed efficiency and digestibility of red sea bream (Pagrus major). Aquaculture, v.250, p.666-673, 2005.

BISWAS, A.K.; SEOKA, M.; TANAKA, Y.; TAKII, K.; KUMAI, $H$. Effect of photoperiod manipulation on the growth performance and stress response of juvenile red sea bream (Pagrus major). Aquaculture, v.258, p.350-356, 2006.

BISWAS, A.K.; TAKEUCHI, T. Effect of different photoperiod cycles on metabolic rate and energy loss of both fed and unfed adult tilapia Oreochromis niloticus: Part II. Fisheries Science, v.68, p.543-553, 2002.

BISWAS, A.K.; TAKEUCHI, T. Effect of photoperiod and feeding interval on food intake and growth rate of Nile tilapia Oreochromis niloticus L. Fisheries Science, v.69, p.1010-1016, 2003.

BROMAGE, N.; PORTER, M.; RANDALL, C. The environmental regulation of maturation in farmed finfish with special reference to the role of photoperiod and melatonin. Aquaculture, v.197, p.63-98, 2001.

DOWNING, G.; LITVAK, M.K. Effects of light intensity, spectral composition and photoperiod on development and hatching of haddock (Melanogrammus aeglefinus) embryos. Aquaculture, v.213, p.265-278, 2002.

EL-SAYED, A.F.M.; KAWANNA, M. Effects of photoperiod on the performance of farmed Nile tilapia Oreochromis niloticus. I-Growth, feed utilization efficiency and survival of fry and fingerlings. Aquaculture, v.231, p.393-402, 2004.

FAO. Fisheries and aquaculture information and statistic service: 2007: aquaculture production: 1950-2006: FISHSTAT Plus: universal software for fishery statistical time series. Disponível em: $<$ http://www.fao.org/fi/statist/FISOFT/FISHPLUS.asp> Acesso em: 20 jul. 2007.

GIRI, S.S.; SAHOO, S.K.; SAHU, A.K.; MOHANTY, S.N.; MUKHOPADHYAY, P.K.; AYYAPPAN, S. Larval survival and growth in Wallago attu (Bloch and Schneider): effects of light, photoperiod and feeding regimes. Aquaculture, v.213, p.151-161, 2002.

HAN, D.; XIE, S.; LEI, W.; ZHU, X.; YANG, Y. Effect of light intensity on growth, survival and skin color of juvenile Chinese longsnout catfish (Leiocassis longirostris Günther). Aquaculture, v.248, p.299-306, 2005.

IMSLAND, A.K.; FOLKVORD, A.F.; STEFFANSSON, S.O. Growth, oxygen consumption and activity of juvenile turbot 
(Scophthalmus maximus) reared under different temperatures and photoperiods. Netherlands Journal of Sea Research, v.34, p.149-159, 1995.

IMSLAND, A.K.; FOSS, A.; FOLKVORD, A.; STEFANSSON, S.O.; JONASSEN, T.M. Genotypic response to photoperiod treatment in Atlantic cod (Gadus morhua). Aquaculture, v.250, p.525-532, 2005.

IMSLAND, A.K.; FOSS, A.; STEFANSSON, S.O.; MAYER, I.; NORBERG, B.; ROTH, B.; JENSSEN, M. Growth, feed conversion efficiency and growth heterogeneity in Atlantic halibut (Hippoglossus hippoglossus) reared at three different photoperiods. Aquaculture Research, v.37, p.1099-1106, 2006.

KUBITZA, F. Tilápia: tecnologia e planejamento na produção comercial. Jundiaí: F. Kubitza, 2000. 285p.

MENDES, P.P. Estatística aplicada à aquiicultura. Recife: Bagaço, 1999. 265p.

MEURER, F.; HAYASHI, C.; BOSCOLO, W.R.; SCHAMBER, C.R; BOMBARDELLI, R.A. Fontes protéicas suplementadas com aminoácidos e minerais para a tilápia-do-nilo durante a reversão sexual. Revista Brasileira de Zootecnia, v.34, p.1-6, 2005.

PEZZATO, L.E.; BARROS, M.M.; FRACALOSSI, D.M.; CYRINO, J.E.P. Nutrição de peixes. In: CYRINO, J.E.P.; URBINATI, E.C.; FRACALOSSI, D.M.; CASTAGNOLLI, N. Tópicos especiais em piscicultura de água doce tropical intensiva. São Paulo: TecArt, 2004. p.75-169.

PORTER, M.J.R.; RANDALL, C.F.; BROMAGE, N.R.; THORPE, J.E. The role of melatonin and the pineal gland on development and smoltification of Atlantic salmon (Salmo salar) parr. Aquaculture, v.168, p.139-155, 1998.

RAD, F.; BOZAOGLU, S.; GÖZÜKARA, S.E.; KARAHAN, A.; KURT, G. Effects of different long-day photoperiods on somatic growth and gonadal development in Nile tilapia (Oreochromis niloticus L.). Aquaculture, v.255, p.292-300, 2006.

REYNALTE-TATAJE, D.; LUZ, R.K.; MEURER, S.; ZANIBONI-FILHO, E.; NUÑER, A.P.O. Influência do fotoperíodo no crescimento e sobrevivência de pós-larvas de piracanjuba Brycon orbignyanus (Valenciennes, 1849) (Osteichthyes, Characidae). Acta Scientiarum, v.24, p.439-443, 2002.

SIGHOLT, T.; STAURNES, M.; JAKOBSEN, H.J.; ÅSGÅRD, T. Effects of continuous light and short-day photoperiod on smolting, seawater survival and growth in Atlantic salmon (Salmo salar). Aquaculture, v.130, p.373-388, 1995.

TRIPPEL, E.A.; NEIL, S.R.E. Effect of photoperiod and light intensity on growth and activity of juvenile haddock (Melanogrammus aeglefinus). Aquaculture, v.217, p.633-645, 2003.

TROTTER, A.J.; BATTAGLENE, S.C.; PANKHURST, P.M. Effects of photoperiod and light intensity on initial swim bladder inflation, growth and post-inflation viability in cultured striped trumpeter (Latris lineata) larvae. Aquaculture, v.224, p.141-158, 2003.

ZIMMERMANN, S. O bom desempenho das chitraladas no Brasil. Panorama da Aquiicultura, v.10, p.15-19, 2000.

Recebido em 6 de novembro de 2007 e aprovado em 3 de junho de 2008 